\title{
Physics-Based Modeling of the Pregnant Woman
}

\author{
Juan Pablo de la Plata Alcalde, Lazar Bibin, Jérémie Anquez, \\ Tamy Boubekeur, Elsa Angelini, and Isabelle Bloch \\ Télécom ParisTech, CNRS UMR 5141 LTCI, Signal and Image Dept, \\ Paris, France \\ \{delaplat, lazar.bibin, jeremie. anquez, tamy. boubekeur, \\ elsa.angelini, isabelle.bloch\}@telecom-paristech.fr \\ http://www.tsi.enst.fr
}

\begin{abstract}
High quality obstetric images have become readily available with fast magnetic resonance imaging protocols or new 3D ultrasound probes. These obstetric images provide detailed information which can be used to create realistic models of the fetus at different stages of gestation. Unfortunately, the anatomy of the mother is only partially visible in the obstetric images and a full model of the pregnant woman can only be based on deformations of a non-pregnant woman body. Numerical pregnant women models are useful for a variety of applications, including dosimetry studies to assess the potential health effects of low-frequency electro-magnetic fields generated by power lines and electrical devices, planning of delivery procedure or training of obstetrical echographers. Only few models of pregnant women have been developed up to now, and they all lack realism and medical accuracy in representing the different stages of gestation and the anatomical variability of the fetal anatomy and positioning. This paper describes a novel methodology to create a set of detailed 3D pregnant women based on a series of controlled automatic deformations of a generic woman body envelope to insert pre-segmented utero-fetal units using a physics-based interactive modeler.
\end{abstract}

Keywords: Anatomical modeling, medical simulation, fetus, ante-natal imaging.

\section{Overview of Existing Pregnant Women Models}

Numerical models of the adult and infant body used for dosimetry [1] can be categorized into three classes, depending on the type of representation. The first class includes mathematical models, where anatomical structures are described using surface equations. They were proposed for their ease of implementation in pioneer works [2], but they are inherently limited in terms of anatomical realism. For instance, modeling the brain with an ellipsoid remains a coarse simplification of the organ. The second class includes voxelized models, built with segmented medical images, which are now preferred as they accurately represent the human anatomy. However, medical data is not always available and its segmentation requires an important amount of manual interaction. This explains the introduction of a third class: the synthetic models, built using computer graphics

F. Bello and S. Cotin (Eds.): ISBMS 2010, LNCS 5958, pp. $71+81,2010$.
(C) Springer-Verlag Berlin Heidelberg 2010 
software tools such as Blender 3]. The most satisfying models are generated under supervision of experienced anatomists, to ensure their realism [4.

In its 2005 research agenda, the World Health Organization stressed out the need for developing numerical models of the pregnant woman at different stages of pregnancy, in order to study the influence of electromagnetic fields on the fetus. Since whole body medical imaging data sets cannot be acquired on a pregnant patient for ethical reasons (unnecessary fetal exposure) and technical limitations (long acquisition time), two strategies have been employed to build such models. The first strategy consists in elaborating truncated voxelized models. For example, CT images were used to generate models of the maternal trunk between the 12 th and the 36th weeks of gestation in [5], distinguishing the uterus, the amniotic fluid, the fetal soft tissues and the fetal bones within the utero-fetal-unit models.

However, whole body models, i.e. representing the pregnant woman from headto-tow, are desirable and constitute the second modeling strategy. The few works dedicated to model the entire pregnant woman at different stages of pregnancy are based on emerging hybrid modeling, which relies on the combination of mathematical, voxelized and/or synthetic models. In [6], a set of mathematical models of the utero-fetal unit (UFU) at 8, 13, 26, and 38 weeks of gestation 7] were voxelized and embedded in the non-pregnant voxelized model NAOMI. Voxel editing was required to translate and deform NAOMI organs away from the uterus. In 8, hybrid models using the UFU and maternal organs models from [9], the VIP-MAN model from [10] and a synthetic model of the fetus surface were merged to construct 3D surface models of a pregnant woman with detailed organs. Three models were built at 3, 6, and 9 months of pregnancy. To insert the UFU, the maternal organs were manually translated and deformed to avoid overlaps using free-form deformation lattices. Fetal soft tissues, brain and skeleton were distinguished. In [11, a voxelized model of the UFU at 26 weeks of gestation was extracted from magnetic resonance imaging (MRI) data. UFU models at 13 and 18 weeks of gestation were obtained by downscaling. The models are then embedded inside a detailed voxelized non-pregnant woman model 12 using free-form deformations.

Our group has proposed a methodological framework in 1314 to create UFU models from medical images and generate hybrid pregnant woman models. Several realistic and detailed voxelized models of the UFU were built using MRI and 3D ultrasound (US) data sets, covering the whole pregnancy. Insertion and placement of the UFU into a woman body model was initially performed manually. Automating this task is desirable to enable the generation of multiple models, while limiting manual and subjective interactions.

In this paper, we propose a method to automatically insert the UFU into a synthetic woman body, which is deformed to host the whole uterus. As dosimetry or medical studies may require models represented in a reclined or standing positions, we also want to simulate different positioning configurations, while medical image acquisitions are always performed with patients lying down. To guarantee the realism of our women body models as well as the representation of 
the major variations in anatomical configurations and morphologies, we decided to design a tool to control the fat distribution on the pregnant woman envelope.

\section{Utero-Fetal Modeling}

\subsection{Image Database}

With the collaboration of obstetricians from the hospitals of Port-Royal and Beaujon (Paris, France), we gathered 18 3D ultrasound (US) sequences between 8 and 14 WA with high image quality. These US images have a sub-millimetric and isotropic resolution (typically $0.6 \times 0.6 \times 0.6 \mathrm{~mm}^{3}$ ).

In collaboration with pediatric radiologists from the Saint Vincent de Paul hospital (Paris, France), we gathered 22 MRI Steady State Free Precession (SSFP) sequences [15] between 26 and 34 WA. Strict image quality criteria for inclusion in the database are: large field of view to include the whole uterus, good overall contrast, good spatial resolution $\left(1 \times 1 \times 4 \mathrm{~mm}^{3}\right)$, fast acquisition (less than 30 seconds), and low sensitivity to fetal movement artifacts.

\subsection{Image Segmentation}

The medical images used to build the UFU models are rather novel and have not yet lead to much work in the image processing community. Therefore, we developed specific tools for their segmentation, combining automatic methods for some tissues and interactive refinements, using the software tools MIPAV (http://mipav.cit.nih.gov/) and Slicer (www.slicer.org).

Regarding the 3D US data, statistical distributions of the tissues were integrated in a deformable model to differentiate automatically the amniotic fluid from the fetal and maternal tissues 16 .

For the 3D MRI data, the segmentation procedure is based on shape and appearance models and deformable contours [17. It was performed automatically for some fetal organs (eyes, brain, ...) and semi-interactively for some others.

These approaches have been validated by our clinical collaborators, who provided the data and work routinely with them to precisely measure fetal growth of individual organs. This validation was performed visually on a number of representative images (both US and MRI ones). This type of validation was considered as sufficient for the targeted application, where a very high accuracy is not mandatory to achieve a good realism of the derived models.

\subsection{UFU Surface Reconstruction}

To reconstruct the surface mesh of the tissues from the segmentation results of the 3D US images we used a standard marching cubes algorithm. Nevertheless, using this method with the segmentation results of the MRI data provided meshes with staircase effects. To reconstruct smooth meshes, we used multiple point-based graphics tools, in particular the moving least square (MLS) operator, which has recently emerged as an efficient and robust technique in digital 
geometry processing [13]. All the UFU models were anatomically validated by obstetricians and pediatric physicians.

\section{Physics-Based Modeling of Pregnant Women}

The developed pregnant women models are composed of three components: (i) a set of pre-segmented UFU, (ii) an empty woman body envelope provided by Daz Studio 18, called Victoria, (iii) the pelvis bone of a pregnant woman, presegmented on a CT data set of a subject having the same height as Victoria $(167 \mathrm{~cm})$, provided in 5]. The goal is to apply physics-based simulations on the woman body to create realistic and flexible heterogeneous models of pregnant women. We first prepare the models to use them efficiently while running the simulations. Second, we place the models in the right position and finally, we run the simulation to get the final woman models, which can also be interactively sculpted, using physics-based methods, to add a layer of fat.

\subsection{Generation of Multimodel Representations}

We have chosen the open source medical simulation framework SOFA 19] to perform the physics-based pregnant women body simulations. The SOFA architecture relies on several innovative concepts, in particular the notion of multimodel representations. The simulation components consist of the woman body envelope, the skeleton and the UFU witch are defined with three types of representations (visual, behavior and collision) to respectively optimize rendering, deformation and collision detection tasks.

We use the original surface models of the three components for the visual representation and create new models for the behavior and collision representations:

1. For the skeleton, we do not generate behavior nor collision representations since we only use it as a visual landmark for positioning the UFU.

2. Victoria's body envelope behavior representation is generated from a sparse free-form deformation (FFD) of the bounding box of the visual model's trunk. We focus on the deformations of the abdominal wall, considering that the head, arms and legs do not deform during the simulation. Only the FFD cells containing some matter are considered to generate an hexahedral finite element model (FEM) of a force field while the mass is uniformly distributed.

3. The UFU is considered as a rigid object with six degrees of freedom (3D translation and $3 \mathrm{D}$ rotation). It is moved in space using the motion data provided by a Biovision Hierarchy file [20]. For the collision representation between the UFU and the woman envelope, the original models are decimated, which strongly simplifies contact detection computation.

Finally, each representation is connected to each other using standard mappings.

In the following, the segmented fetus will be considered as a rigid structure. Although this is obviously not the case, this assumption allows us to build models 
where the position and shape of the fetus match the ones viewed in the acquired images, thus guaranteeing the realism and the fidelity between the images and the proposed models.

\subsection{Positioning of Anatomical Elements within the Woman's Body}

Victoria's representations are positioned in standing positions inside the simulation scene. The skeleton is placed inside Victoria's body interactively, using the free software tool Blender [3]. Three landmark points are manually selected on the skeleton of the generic woman body: the two femoral head centers and the center of the vertebral disk between the L3 and L4 lumbar vertebra (see Fig. 1). The three corresponding points are identified on the MRI images used to segment the UFU, enabling a rigid registration of the UFU collision and visual representations inside Victoria's body. Figure 2 shows four UFU automatically placed in different women models.

\subsection{Interactive Pregnant Woman Body Sculpting Tool}

An interactive body sculpting tool was designed to model the fat layer on Victoria's body. The idea is to consider that the standard $3 \mathrm{D}$ woman model is composed of two different layers of skin and fat. The volume of the fat layer is interactively modified using a mouse pointer. The points of the surface under the pointer are pushed up towards the normal direction by applying a constant force balanced by the Wendland kernel, as in (1). The force applied on the surface is modeled with a radial basis function, centered on the $3 \mathrm{D}$ projection of the mouse cursor onto the surface and vanishing within a prescribed Euclidean distance, as detailed in (2). In practice, we use the monotically decaying Wendlend quartic kernel 21].

$$
\omega(x)= \begin{cases}\left(1-\frac{x}{h}\right)^{4}\left(4 \frac{x}{h}+1\right) & \text { if } x<h \\ 0 & \text { otherwise. }\end{cases}
$$

The surface $\mathcal{S}$ is sampled by the mesh $\mathcal{M}=\{\mathcal{V}, \mathcal{F}\}$ where $\mathcal{V}=\left\{v_{i}\right\}$ are the vertex and $\mathcal{F}$ the faces. In our case, the following displacement field is applied on $\mathcal{M}$ :

$$
\forall v_{i} \in \mathcal{V}, f\left(p_{v_{i}}\right)=\alpha \omega\left(q-p_{v_{i}}\right) n_{v_{i}} .
$$

where $q$ is the point of the surface $\mathcal{S}$ under the mouse cursor, $p_{v_{i}}$ is the 3D position of $v_{i}, h$ is an user defined support radius, $n_{v_{i}}$ is the normal to the surface $\mathcal{S}$ at vertex $p_{v_{i}}$ and $\alpha$ is a user defined weight.

The sculpting process is operational when the mouse cursor is positioned at a point location $q$ on Victoria and any mouse button is clicked. The displacement field is applied, until the button is released, on the surface points $p_{i}$ that are inside the circle centered at point $q$ and with radius $h$. The displacement field decreases away from the center point $q$.

Once the fat layer has been modified, the volume encapsulated between the skin and the fat layers (considered as new an independent closed surfaces) is 


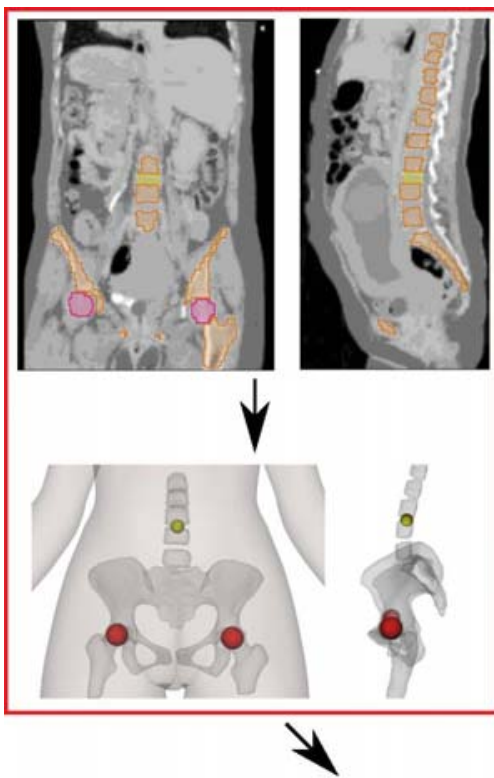

(a)

(b)

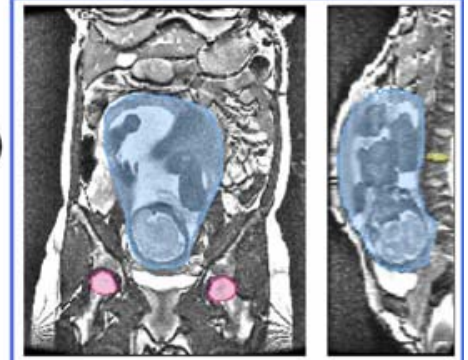

(c) (d)

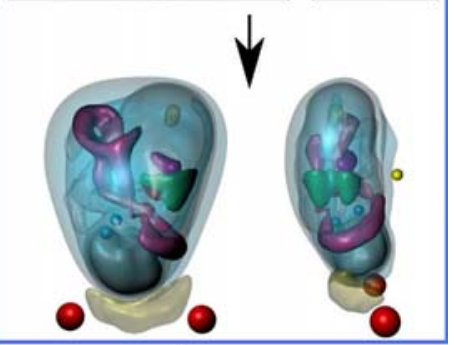

(e)
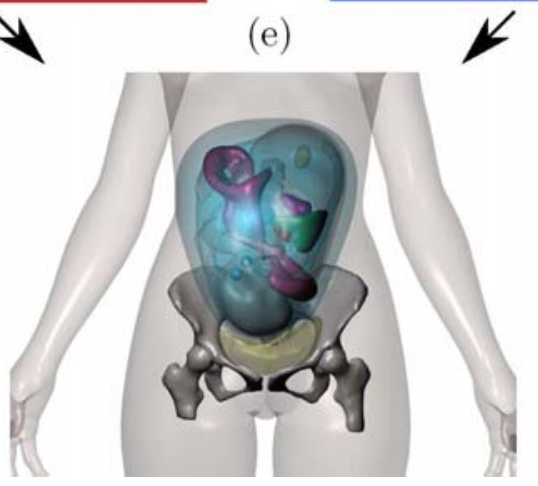

Fig. 1. Insertion of the UFU inside Victoria's body envelope. (a) Segmentation of the skeleton in orange, the femoral heads in red, and the vertebral disk between L3 and L4 in yellow from CT data. (b) Coronal and sagittal views of the reconstructed skeleton and landmark points inserted in Victoria's body. (c) Segmentation of the uterus in blue, the femoral heads in red, and the vertebral disk between L3 and L4 in yellow from from MRI data. (d) Coronal and sagittal views of the reconstructed UFU and landmark points. (e) Final positioning of the UFU. In the red frame, the landmark points identification process is performed one time, whereas in the blue frame the process is performed for each new UFU to insert, generating individual models.

filled with tetrahedras using the Isosurface Stuffing Algorithm [22. Then, a corotational FEM force field is defined to determine its behavior. Points where there is no fat accumulation can also be defined using the mouse. Figure 3 shows with red dots the areas without fat development.

At these point locations, the force applied is always set to zero so that skin and fat layers remain attached together, creating a barrier to the fat expansion 


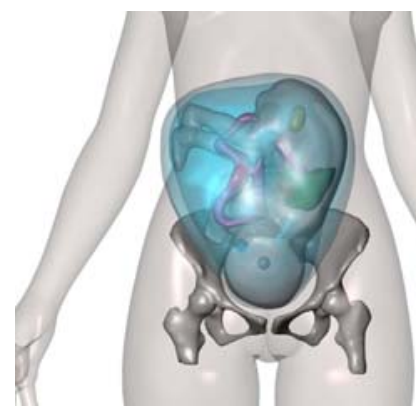

(a)

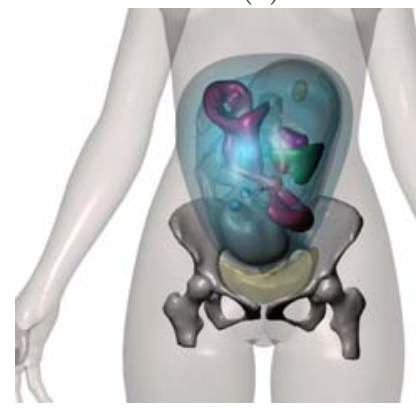

(c)

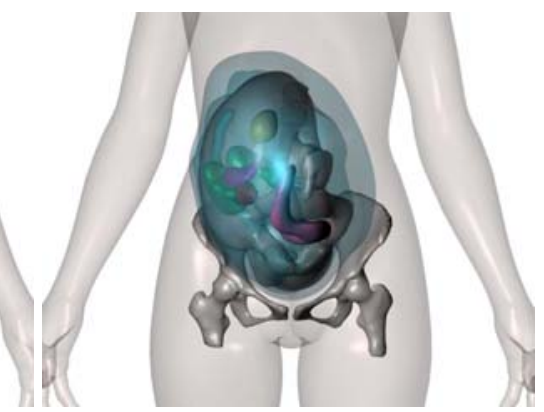

(b)

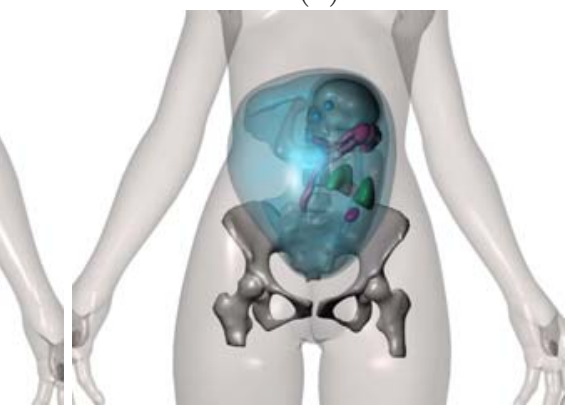

(d)

Fig. 2. Four different UFU positioning results using three landmark points. (a) Pregnant woman in supine position. (b) Pregnant woman in lateral position. (c) Pregnant woman with a filled bladder. (d) Pregnant woman with a fetus in breach position.
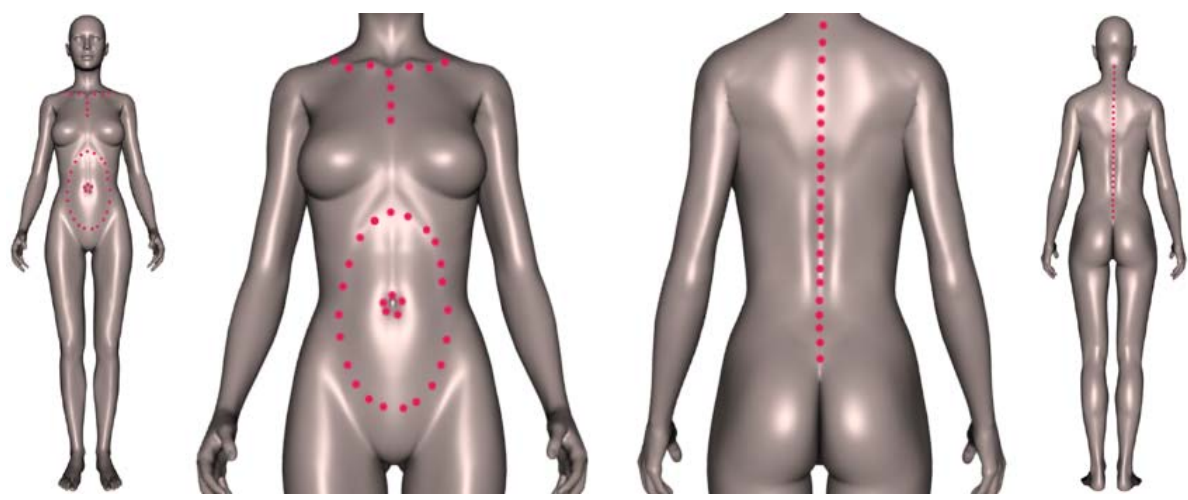

Fig. 3. Body regions without fat accumulation represented by dotted red lines

and accumulation. This procedure is necessary to delimit fat "pockets" and prevent un-physiological fat diffusion under gravitational effects. Figure 4 shows the added fat layers represented by a set of tetrahedras. 


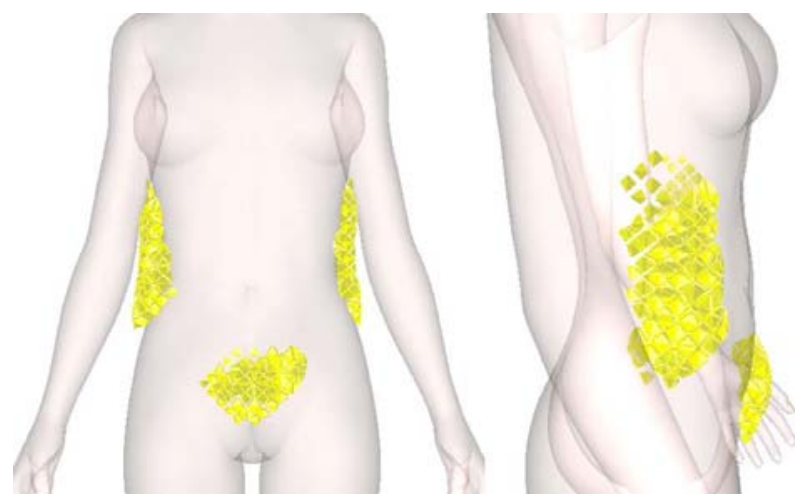

Fig. 4. Set of tetrahedras representing the fat layers

\subsection{Simulations Running}

The UFU orientation is affected by the laying down position of the mother during the imaging examination. To represent the same mother in a standing position, we need to apply a pendulous movement on the UFU. We first apply a rigid 6 degrees of freedom transformation to align skeleton points of references, as described previously (see Fig. (5). Then, we constrain its position and its rotation

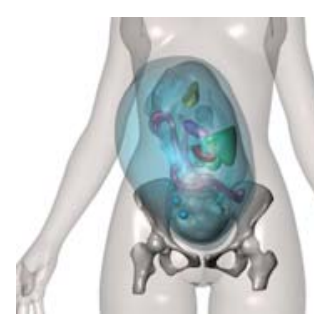

(a)

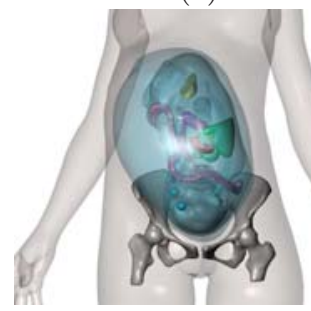

(c)

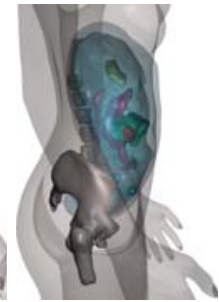

(b)

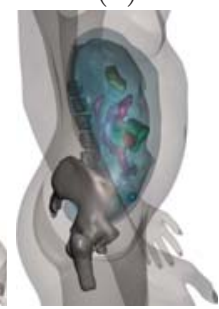

(d)

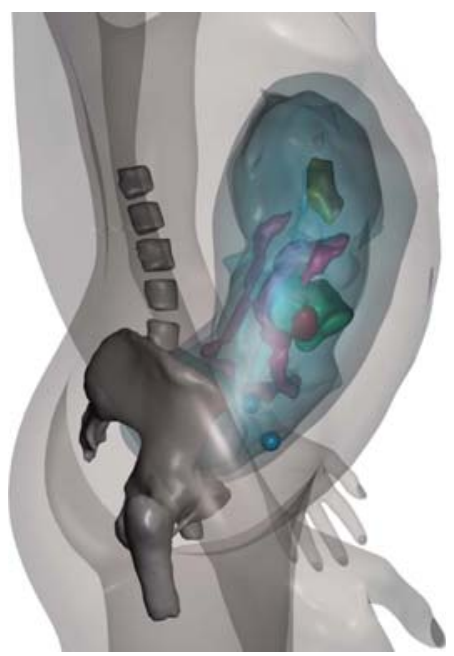

(e)

Fig. 5. Deformations of the Victoria's body envelope to include the UFU: (a,b) UFU positioning without collision detection in coronal and sagittal views. (c,d) UFU positioning using collision detection in coronal and sagittal views. (e) Deformed body of Victoria's body to simulate a standing position, after UFU rotation. 
except for a rotation around an axis defined by the two femoral heads, which enables the UFU to rotate while pushing the abdominal walls. We decided to apply a rotation of 35 degrees to the UFU according to our medical experts, using again the Biovision Hierarchy file [20] (see Fig. [5). The coarse model created for the collision detection is used to displace the abdominal wall away from the rotating UFU. At the same time, the added fat layer deforms under the gravitational force field effect, deforming the overall envelope model.

As for the validation of the whole models, it has been performed by our clinical collaborators, who could in particular estimate the accuracy of the positioning of landmark points and the realism of the reconstruction. It should be moreover noticed that the proposed automated positioning method keeps the distance between the skin and the uterus wall constant, thus again guaranteeing the fidelity with the acquired imaging data.

\section{Discussion and Conclusion}

In this paper, we have proposed a novel automated methodological framework to insert utero-fetal units, segmented on medical images, into a synthetic woman body envelope, which is deformed to host the whole uterus. As dosimetry or medical studies may require models represented in a reclined or standing positions, we also addressed the problem of simulating different positioning configurations. The realism of the UFU was guaranteed by the large database of medical image data sets acquired during this project, covering the whole pregnancy. To guarantee the realism of our women body models as well as the representation of the major variations in anatomical configurations and morphologies, we designed a tool to control the fat distribution on the pregnant woman envelope. Some issues remain regarding the parametrization of such physics-based modeling tool. Indeed, setting the right values for the mass of the fetus and the woman body is a complex task. Finding the right elasticity of the abdominal walls is also difficult to define. For the moment, these parameters are set empirically. Setting all the points where there is no fat needs advanced medical knowledge, and still has to be performed manually. Finally, detailed segmentation of the 3D US and MRI imaging data remains also difficult and still requires manual refinements to include details on the fetal organs.

All our models were anatomically validated by medical experts using visual inspection for the segmentation of the UFU tissues, the insertion of the UFU into the synthetic maternal body, the simulation of the standing position, and the addition of a user defined fat layer.

In conclusion, the proposed approach achieves a significant step further towards hybrid pregnant woman modeling, by proposing both a method to automatically position the fetus inside a generic woman body, and a novel body sculpting tool to simulate fat layers. This allows now studying the influence of the fat thickness for various applications such as dosimetry simulation studies. 


\section{References}

1. Wiart, J., Hadjem, A., Wong, M.F., Bloch, I.: Analysis of RF exposure in the head tissues of children and adults. Phys. Med. Biol. 53, 3681-3695 (2008)

2. Cristy, M., Eckerman, K.F.: Specific absorbed fractions of energy at various ages from internal photon sources. Oak Ridge National Laboratory Report ORNL/TM8381/V1-V7 (1987)

3. Blender, http://www.blender.org/

4. Human Anatomy, http://www.3dscience.com/3D_Models/Human_Anatomy/index.php/

5. Angel, E., et al.: Radiation dose to the fetus for pregnant patient undergoing multidetector CT imaging: Monte Carlo simulations estimating fetal dose for a range of gestational age and patient size. Radiology 249(1), 220-227 (2008)

6. Dimbylow, P.: Development of pregnant female, hybrid voxel-mathematical models and their application to the dosimetry of applied magnetic and electric fields at 50 Hz. Phys. Med. Biol. 51(10), 2383-2394 (2006)

7. Chen, J.: Mathematical models of the embryo and fetus for use in radiological protection. Health Phys. 86(3), 285-295 (2004)

8. Xu, X.G., Taranenko, V., Zhang, J., Shi, C.: A boundary-representation method for designing whole body radiation dosimetry models: pregnant females at the ends of three gestational periods RPI-P3, -P6 and -P9. Phys. Med. Biol. 52(23), 7023-7044 (2007)

9. Shi, C., Xu, X.G.: Development of a 30-week-pregnant female tomographic model from computed tomography (CT) images for Monte Carlo organ dose calculations. Med. Phys. 31, 2491-2497 (2004)

10. Xu, X.G., Chao, T.C., Bozkurt, A.: VIP-MAN: an image-based whole-body adult male model constructed from color photographs of the visible man project for multi-particle Monte Carlo calculations. Health Phys. 78(5), 476-486 (2000)

11. Nagaoka, T., Saito, K., Takahashi, M., Ito, K., Watanabe, S.: Anatomically realistic reference models of pregnant women for gestation ages of 13, 18, and 26 weeks. IEEE Eng. Med. Biol. Soc., 2817-2820 (2008)

12. Nagaoka, T., Watanabe, S., Sakurai, K., Kuneida, E., Taki, M., Yamanaka, Y.: Development of realistic high-resolution whole-body voxel models of Japanese adult males and females of average height and weight, and application of models to radiofrequency electromagnetic-field dosimetry. Phys. Med. Biol. 49, 1-15 (2004)

13. Anquez, J., Boubekeur, T., Bibin, L., Angelini, E., Bloch, I.: Utero-fetal unit and pregnant woman modeling using a computer graphics approach for dosimetry studies. In: Medical Image Computing and Computerized Assisted Intervertion (MICCAI), London, UK. LNCS, vol. 5761, pp. 1025-1032. Springer, Heidelberg (2009)

14. Bibin, L., Anquez, J., Angelini, E., Bloch, I.: Hybrid 3D pregnant woman and fetus modeling from medical imaging for dosimetry studies. International Journal of Computer Assisted Radiology and Surgery (2009)

15. Anquez, J., Angelini, E., Bloch, I., Merzoug, V., Bellaiche-Millischer, A., Adamsbaum, C.: Interest of the Steady State Free Precession (SSFP) sequence for 3D modeling of the whole fetus. IEEE Eng. Med. Biol. Soc., 771-774 (2007)

16. Anquez, J., Angelini, E., Bloch, I.: Segmentation of fetal 3D ultrasound based on statistical prior and deformable model. In: IEEE International Symposium on Biomedical Imaging (ISBI), pp. 17-20 (2008)

17. Anquez, J., Angelini, E., Bloch, I.: Automatic segmentation of head structures on fetal MRI. In: IEEE International Symposium on Biomedical Imaging (ISBI), pp. 109-112 (2009) 
18. Daz 3D Studio, http://www.daz3d.com/

19. Allard, J., Cotin, S., Faure, F., Bensoussan, P.J., Poyer, F., Duriez, C., Delingette, H., Grisoni, L.: SOFA - an Open Source Framework for Medical Simulation. Medecine Meets Virtual Reality (MMVR'15), pp. 13-18 (2007)

20. Biovision Hierarchy, en.wikipedia.org/wiki/Biovision_Hierarchy

21. Wendland, H.: Piecewise polynomial, positive definite and compactly supported radial functions of minimal degree. Adv. Comput. Math. 4(4), 389-396 (1995)

22. Labelle, F., Shewchuk, J.R.: Isosurface Stuffing: Fast Tetrahedral Meshes with Good Dihedral Angles. ACM SIGGRAPH 26(3) (2007) 